\title{
COVID-19 pharmacy student perceptions: pharmacists' impact during the COVID-19 pandemic
}

\author{
Bryan Zhang ${ }^{1, *}$, Justina Refela ${ }^{1}$, Frank Breve ${ }^{1}$, Peter Magnusson ${ }^{2,3}$, Joseph Pergolizzi ${ }^{4}$
}

${ }^{1}$ Department of Pharmacy, Temple University, Philadelphia, PA 19140, USA ${ }^{2}$ Centre for Research and Development, Region Gävleborg/Uppsala University, 80320 Gävle, Sweden

${ }^{3}$ Department of Medicine, Cardiology Research Unit, Karolinska Institutet, 11153 Stockholm, Sweden

${ }^{4}$ Clinical Research, NEMA Research Inc., Naples, FL 34108, USA

\section{*Correspondence}

bryan.zhang@temple.edu

(Bryan Zhang)

\begin{abstract}
Pharmacists around the world are playing an active role in educating the public about COVID-19. First-year pharmacy students taking a healthcare course at Temple University in Philadelphia were asked to write papers based on the nature of a pharmacist's contributions during the pandemic. $90 \%$ of the essays outlined the importance of community pharmacists, as they are the most accessible healthcare professional, providing immediate social interaction and drug expertise. They help minimize the need for hospital visits, limiting their time in public, therefore reducing their risk of becoming infected. For the pharmacies that have a drive-thru window, over-the-counter medications and other necessities can be sold to decrease the likelihood of transmission. Many students also proposed the opportunity for more leniency in prescribing power. Unless pharmacists are within a collaborative practice agreement, they cannot prescribe even maintenance medication. Due to the pandemic, it has become increasingly hard to reach the doctor or see them in person, as most offices have switched to telehealth. Clinical pharmacists operating out of hospitals have the opportunity to oversee proposed treatment options or experimental drugs, such as remdesivir. Retail pharmacies are emerging as key resources in this pandemic, and it is important that students see this as a vibrant and important form of pharmacy practice.
\end{abstract}

\section{Keywords}

COVID-19; Pandemic; Pharmacy; Pharmacists; Students; Industry; Community; Hospital

\section{Introduction}

Pharmacists around the world play an active role in educating the public about COVID-19 [1]. As the world mobilizes limited resources to combat the infection, legal extensions have been granted to pharmacists regarding preparing disinfectants, renewing prescriptions for chronic diseases, screening for COVID-19, and possibly in the future administering the COVID-19 vaccination. To manage potential drug shortages, pharmacists may also be allowed to recommend alternative drugs, alternate doses, or compounding formulations [2]. Particularly challenging is the fact that the COVID-19 pandemic was accompanied by an "infodemic" or avalanche of information that spread rapidly by social media. The infodemic can be overwhelming to many people. Added to that, some of the information changed over time with further investigation and other information was not accurate to begin with [3]. More than $90 \%$ of Americans live within five miles of a community pharmacy, and community pharmacists serve on the frontlines of delivering public health information, administering vaccinations, doing point-of-care testing, and facilitating patient interactions with the healthcare system [4]. All of these functions emerged as vitally important during COVID-19.
Pharmacy students were asked to formulate their opinions and ideas on the role of pharmacists in the COVID-19 pandemic and their impact on patients during the COVID-19 pandemic. Using a short essay format, pharmacy students shared their insights as to how different pharmacy settings might most effectively contribute to patient care and public health in these unprecedented times by observing their work and learning environments. They would compile their thoughts into a short 2-page essay and offer their opinions on the matter as well as how this catastrophe could prove to be an opportunity for pharmacists to further their responsibilities by exercising the knowledge attained from their academic years. As aspiring pharmacists, this project allowed them to voice their ideas on what they could possibly advocate for when it is their time to become healthcare workers. The field of pharmacy is everexpanding and it is imperative that students embarking on the working world understand the impact they have in their workplace, wherever they decide to branch out into. This project hopes to compile such information as a foundation for students to grow upon. 


\section{Methods}

165 first-year pharmacy students enrolled in a health care course at Temple University School of Pharmacy in Philadelphia, PA were given an opportunity to prepare a short essay to present their insight on the COVID-19 pandemic and how pharmacists' efforts may contribute to patient care and public health. The opportunity was offered to students as extra-credit and 116 students participated, producing a response rate of $70.3 \%$. The context of the study was formed considering the recent unprecedented events developed by the COVID-19 pandemic. Professor Dr. Breve tasked his students to observe changes within students' work environments and academic surroundings to evaluate the role that pharmacists have in the overall health system and what they could do to expand upon their responsibilities to further their contributions to the worldwide catastrophe. Participants were asked to pick one or two types of pharmacy settings (community pharmacy, hospital setting, compounding pharmacy, industrial setting, pharmacy schools, etc.) and then describe how they might contribute positively to patient care in the era of COVID-19. All 116 essays were read through once to categorize each into specific pharmacy perspectives. In other words, if a student wrote their essay concerning the impact that their community pharmacist had in the pandemic, the authors would categorize the essay into the "community" perspective. If a student wrote about 2 different perspectives such as community and hospital, their essay would tally into both categories. Such cases would explain why there would be more perspectives than the number of essays written. Most students wrote about the impact pharmacists had in the community, hospital, compounding, and industry setting. So, the authors created 5 categories to sort the essays in with the "other" category being a miscellaneous classification that could encompass fields that did not directly fit into the majority of settings. After categorization, two authors each analyzed 58 essays to extract more information regarding the contents of how students approached the research question. There was never disagreement on categorization of an essay since each essay was evaluated by one author. Essay length was left open to the students who embarked on the task. They could write as much or as little as they wanted. However, the university tasked its students to at least write 1000 words worth, which was reflective in most students' essays. Students were not informed that their essays would be used for the purpose of the study. The professor of the health care class made this an optional task for their students in light of current world circumstances and used "extra credit" as an incentive. After the papers were submitted and the credit had been awarded, he believed that the papers could be used to develop a thoughtful research project. He selected two students that had an interest in learning more about COVID-19 and gave them the opportunity to develop a research question out of the essays. The authors did not seek ethical approval as it was not required by the professor supervising the project.

\section{Results}

116 students submitted an essay for review and evaluation. The essays were then separated into one of five perspectives (some essays received more than one tally if the student decided to write about more than one practice setting). See Table 1. Within each perspective, recurrent themes were noted for discussion.

Community pharmacists are frontline healthcare professionals who are easily accessible to the public. Working at retail stores such as CVS, Walgreens, Rite Aid, or local supermarkets such as Giant and ACME, community pharmacists provide the immediate social interaction and drug expertise needed to keep patients healthy and compliant.

In general, community pharmacists can help keep patients with diseases other than COVID-19 adherent to their medications and possibly prevent hospitalizations, which, in turn may reduce hospitalizations and the workload of clinical professionals. It was noted that pharmacists can help minimize the need for too many visits by patients to the pharmacy by providing extended supply days or advocating for early refills. This can help patients reduce the amount of time they need to be out in public, which is generally recommended to reduce the spread of the disease. It was noted by students that community pharmacists can help dispel confusion about drug regimens in COVID-19, for example, whether ibuprofen is safe or should be preferred to acetaminophen. Furthermore, it was noted in the essays that community pharmacists can prove to be a good sound board to facilitate communication with frustrated or confused patients and to get them directed to the right people. One essay recommended that community pharmacies might want to change their hours to accommodate the specific needs of various population groups (older people, working people).

According to some papers, because of the infodemic of COVID-19 "facts", community pharmacists have a significant opportunity to emerge as a resource for patients to help sort through what patients most need to know about COVID-19, its transmission, and its treatment. For instance, vitamin $\mathrm{C}$ has been recommended to boost immunity and, in that way, fight the virus, and community pharmacists may be helpful in advising about that or other over-the-counter remedies for patients interested in improving their overall health in the wake of the pandemic. Even though there is a great deal of relatively confusing information about COVID-19 on social media, one essay recommended that community pharmacists could post information online about COVID-19 and tips for patients to stay safe. Some students related their experience as social media chairs within the school's multiple organizations explaining that "infographics, flyers, and webinars could be a means to spread awareness about the pandemic. The amount of 'likes' and comments justified the post's reach and popularity to people in the community and allowed for safe interaction and communication about the topic". Many students believe that this form of outreach can be extended to pharmacists as many in the field of academia have already held lectures on the pandemic.

To reduce exposure of patients, essays recommended that community pharmacists could pioneer new ways of selling products, such as expedited drive-through service for both prescription and over-the-counter products, acceptance of telephone or electronic orders, and maintaining adequate inventories of hand sanitizer, face masks, and other pandemic supplies. One essay suggested a "mobile pay program in which patients 
TA B L E 1. Most essays (90.5\%) considered the issue of COVID-19 public health support by pharmacists from the community perspective.

\begin{tabular}{|ccccc}
\multicolumn{5}{c}{ Perspectives from different pharmacy settings } \\
Community & Hospital & Compounding & Industry & Other \\
105 & 12 & 1 & 3 & 5 \\
\hline
\end{tabular}

can pay their balance ahead of time to lessen the direct inpharmacy contact". Although this effort has already been implemented at certain pharmacies, like Rite Aid, other community pharmacies could give chase to such an idea to further the safety of their staff.

Community pharmacists have a vital link to physicians and these connections could be broadened to that of an ambulatory pharmacist under the Collaborative Practice Agreement. Pharmacists can suggest therapeutic substitutions to physicians when specific medications are in short supply, provide dosage form changes, as well as dosage strength modifications. A common consensus across a handful of essays was that "pharmacists could be allowed to exercise more freedom over a patient's medication as long as it's within their scope of practice. This would not only reduce the workload of physicians by decreasing the amount of phone calls made during this time, but also develop a stronger patient-care service between the customer and the pharmacist regarding their medication regimens. Physicians can focus more of their efforts on COVID-19-related issues rather than handling phone calls that a pharmacist with more freedom can easily solve". Nevertheless, this means that community pharmacists must sometimes be creative and resourceful during the pandemic to find ways to contact and discuss such matters with prescribers. Many doctors have shut down their offices or have drastically cut office hours, opting instead for telemedicine or abbreviated visits. The pharmacy students suspected that this might lead to prescribing with insufficient medical histories or the prescribing of drug therapies with potential interactions; it was recommended that pharmacists could help identify such potential drug-drug interactions or duplicate therapies.

Several essays brought up the issue of health insurance in the community pharmacy setting and saw community pharmacists as a resource to help determine the best therapeutic choices for the patient based on their individual coverage. Many frail, community-dwelling geriatric patients are enrolled in the Program for All-Inclusive Care of the Elderly (PACE). PACE provides these patients with both Medicare and Medicaid benefits; community pharmacists can help navigate the sometimes complex insurance issues for this group.

Many students agree that although community pharmacists' current responsibilities contribute significantly to having a positive impact, there is always room for further optimization of patient care. For example, students are trained to take vital signs during their 1000 hours at an Accreditation Council for Pharmacy Education (ACPE) program. These skills were recommended in one essay to "triage patients for those who might have COVID-19 versus those who likely had another infection, such as influenza". Regulations currently allow point-of-care testing in many community pharmacies, which students recognized to offload some of the burden placed on testing facilities and hospitals.

One essay advocated that community pharmacists should document and count inventory of COVID-19-related medications in the same manner that they document and inventory Schedule 2 controlled substances, such as oxycodone and morphine. The concept here is that COVID-19 medications must be spared and used only for those with the diagnosis of COVID-19. A student also reasoned that it would provide, "a tighter lock on these medications so that risk of diversion would be minimized", which was an idea reiterated multiple times in other essays. Another novel idea in one of the essays was a rapid-response system designed for emergency distribution of COVID-19 resources within the community. For example, if a hotspot broke out, the community pharmacy could dispense not just related drugs but also over-the-counter products and supplies, such as hand sanitizer, masks, and gloves. Another essay suggested that community pharmacies set up home delivery services to reduce direct contact with patients.

The role of the hospital pharmacy is quite different, in that hospitals deal with the more severe cases of COVID-19 and may be involved in data collection and even clinical trials. In the outpatient setting, pharmacists fill medications and deliver them directly to the patients' rooms prior to discharge so they do not have to leave their homes to get their newly prescribed medications. In the inpatient setting, universal treatment regimens have been distributed to elicit a standard of drug therapy for the levels of severity accompanying the virus. These regimens or charts can be used broadly by all pharmacists as tools to recommend substitutions or alternatives to physicians. Clinical trials testing drugs such as hydroxychloroquine, azithromycin, and remdesivir are largely interpreted and reviewed by pharmacists and other healthcare professionals, regulators, and manufacturers to determine the safety and efficacy of various drugs. Data collected from these trials are crucial to advance the worldwide knowledge of the novel virus. Clinical pharmacists in the hospital setting can also round with physicians to track patients' response to experimental treatments to ascertain the feasibility of a given drug protocol and answer questions about the pharmacological regimen. Thus, the role of the hospital pharmacist is seen as a vital collaborator on an interdisciplinary healthcare team.

Pharmacists in the hospital setting have also been encouraged to limit the exposure of the virus through disinfection cart trays and supplies. Regarding respiratory devices, pharmacists are encouraged to recommend COVID-19 positive patients substitute nebulizers for inhalers, whereas COVID-19 negative patients utilize nebulizers. This decreases the respiratory particle generation and the potential risk of transmission.

Finally, industrial and compounding pharmacies were only briefly mentioned in this exercise but were seen to have a 
key role in the development of the vaccine as well as the deployment of resources to manufacture more COVID-19-related supplies, that is, cleaning products, disinfectants, sanitizers, and so on. Students elaborated that industry pharmacists have an important role in monitoring for adverse drug reactions affiliated with the drugs approved for emergency use such as hydroxychloroquine and remdesivir. Although these drugs had not been FDA approved, pharmacists are tasked with the important job of gathering data from hospitals and researchers to organize safety and efficacy data for future reference. Essays concerning compounding pharmacists discussed their potential role in the distribution of hygienic equipment to the public in which a global pandemic may cause a shortage as well an expansion in their responsibilities to include close monitoring of how healthcare settings could improve conservative use of these supplies.

Students argued that other pharmacist professionals such as professors and educators play an important role in educating other pharmacists about the mechanisms of action of the SARS-CoV-2 virus and how it enters the body, how disease appears to be transmitted, the course of the illness, and its possible sequelae. The same infodemic that can overwhelm patients affects healthcare professionals. Over 2,400 peerreviewed scientific articles were indexed by the databases of the National Institutes of Medicine (PubMed) in the first three months of the pandemic alone [5]. At the end of August 2020, a keyword search for "COVID-19" in PubMed turns up over 44,000 results. Thus, academic pharmacists can help sort through this material and help healthcare professionals navigate this information to find what is most accurate and clinically relevant. These presentations need not be delivered as academic lectures on college campuses, but can be transmitted on the Zoom platform, by social media, podcasts, or other means.

\section{Discussion}

Looking at the results, there is overwhelming data by students describing the community perspective. This may be since the community pharmacy experience is most familiar to first-year pharmacy students or that community pharmacy is on the frontlines of the COVID-19 pandemic. Present in multiple student essays is the emphasis that "pharmacists have always been the most accessible healthcare provider". This is especially true during the pandemic where "other professionals have closed their doors to patients and community pharmacies remained open to the public despite stricter lockdown restrictions" [6]. Retail pharmacies are emerging as key resources in this pandemic, and it is important that students see this as a vibrant and important form of pharmacy practice that can not only help to ease the stress on the healthsystem through their expected responsibilities, but expand their roles to educators and advisors to their customers as they are "the first point of contact to those in need and improve early recognition of mental health conditions". Tying in with the fact that students believe that community pharmacists have the knowledge to provide effective care and a means of social intimacy with their customers, it is becoming more common that a "growing number of community pharmacists are trained in Mental Health First Aid" [7], which can help those in quarantine to seek pharmacists for psychiatric help. Although there are services dedicated to helping those in mental need, pharmacists can act as both the medical distributor and the mental relief to not only help those with declining mental states but decrease the spread of misinformation likely to be showcased on media [8]. Relating to the many students that believed that community pharmacists should be given more freedom to the likes of an ambulatory pharmacist with a Collaborative Practice Agreement with a physician, "many countries have implemented legislative changes to support the evolution of pharmacists' roles during these periods, including pharmacists participating in first-responder and other nontraditional roles with other health care providers" [9]. These increased responsibilities have included medication dispensing, referrals and consultation, chronic disease management, patient education, psychological support for the community, and provision of support for COVID-19 related homecare services [10]. In fact, Poland was noted to have expanded their pharmacists' prescriptive authority as they were "granted the right to prescribe drugs for themselves and their closest relatives" [2] which were similarly reimbursed from public sources as when a physician issues a prescription.

Students who wrote about hospital pharmacy provided insight as to the workflow of pharmacists within a clinical setting. The role of the hospital pharmacist in the COVID19 pandemic is more a role of researcher, collaborator, and advisor to physicians since they would have little to no interactions with patients in the hospital. Key to standardizing treatment regimens, "clinical pharmacists created a rational drug use manual for frontline medical personnel to apply treatment of COVID-19, including usage and dosage, solvents, precautions, adverse drug reactions and dose adjustment for special populations, such as the pregnant women, children, elderly patients, dialysis patients, extracorporeal membrane oxygenation (ECMO) patient, etc.” [11]. Students undergoing courses related to treatment that quite possibly could treat COVID-19 have emphasized the importance that pharmacists have in being involved with testing some of these drugs. Currently, there is no specific drug that has been confirmed to treat COVID-19; and the ones that have shown promise have not been tested to the extent where researchers can rule out any adverse reactions. Therefore, hospital and clinical pharmacists are imperative to assisting the process of making "evidencebased decisions for medications and assist clinicians in formulating and adjusting drug regimens of COVID-19 patients" [12]. Students recognized that community pharmacies often dealt with people who were not infected or had mild cases of COVID-19, quite unlike the work environment for the hospital pharmacists.

Industrial, compounding, and other pharmacy perspectives were touched upon by a few students. Although they did not receive the same amount of attention as community or hospital settings, nonetheless some students made convincing arguments in their essays about how these organizations make important contributions. Using data gathered from clinical trials, industry pharmacies aid in providing the necessary resources and information to manufacturers developing the vaccine for COVID-19. Immunization may be crucial to a return to 
normal for our society as the more people who become immune increases, the likelihood of a decrease in disease spreading. In addition to the development of vaccines, industry pharmacists are integral to progressing information regarding broadspectrum antiviral drugs. Many pharmaceutical organizations who are "engaged to sponsor or conduct clinical trials on vaccines and drugs" need pharmacists to "ensure the appropriate and safe use of medications during the clinical trials". These pharmacists also work closely to maintain pharmacovigilance by tracking information from the "healthcare authorities and other pharmacists dealing with COVID-19 patients about any adverse events of the proprietary drug" [13]. Students also came to a consensus that compounding pharmacies have the necessary assets and skills to manufacture and distribute more sanitizing equipment as their curriculum focuses on this area. In a global pandemic, hand hygiene should be emphasized and utilized by the public to ensure a decrease in the spread of the virus. A study was done to evaluate compounding pharmacists' experience and perceptions on production and utilization of hospital-based alcohol-based hand rub (ABHR) solution for COVID-19 prevention in Addis Ababa. They concluded that many settings indicated an irrational use of sanitizers, which could lead to wastage of products, exposure of healthcare providers to infections and emergence of microbial resistance to alcohol [14]. To combat a global pandemic, students as well as this study recognized the importance that compounding pharmacists can have in maintaining conservative and effective use of these supplies that have a strong potential of being exhausted in these times.

Other pharmacy settings such as teaching positions allow pharmacists to educate future leaders in pharmacy to deal with pandemic events more effectively should they occur in the future. Therapeutics classes and optional lectures available to students have provided anecdotal insight to the experiences that professors have had throughout the pandemic. Learning about how they have adapted as well as evolved through the trials of this unprecedented event can prove to be enlightening to aspiring pharmacists. The experience they provide to their students may prove vital in fighting against current and future viruses. Some programs such as the "Pharmacists as Personalized Medicine Experts (PRIME)" project led by doctors Lisa McCarthy and Beth Sproule in Canada have been "training the next generation of pharmacists towards evidence, informed-based, optimized medication therapy prescription, exposing them to advanced and sophisticated concepts, such as pharmacogenetics and pharmacogenomics". These newly attained skills could expand the role of a pharmacists as a hub for medication information, which can lead to "increasing the health literacy of the community and educating, counseling, and empowering citizens, especially those vulnerable and atrisk groups" [15].

\section{Limitations}

This was an essay assignment offered to a convenience sample of first-year pharmacy students. Data was qualitative.

\section{Conclusions}

In students' opinions, all areas of pharmacy practice can offer significant contributions in dealing with the COVID-19 pandemic. Pharmacists provide valuable services by educating the public and being available as an informational resource, ensuring that optimal treatments are being utilized, becoming involved in testing for infection, with a primary focus on patient safety. Pharmacists, in their own unique way, demonstrate the versatility and spectrum of influences that the profession has not only on routine day-to-day patient care but also on an unexpected crisis. If the world is to overcome this unprecedented pandemic event, then the importance of pharmacists must be realized, and there should be an expansion of their already impactful responsibilities to more flexible roles.

\section{AUTHOR CONTRIBUTIONS}

BZ - Conceived and designed the analysis, Collected and organized the data, Performed the analysis using primary literature, Wrote the paper; JR-Collected and organized the data, Wrote the abstract; FB - Contributed data, Precepted and supervised the main author throughout the publication process, Gave suggestions for revisions of the paper; PM-Precepted and supervised the main author throughout the publication process, Gave suggestions for revisions of the paper; JPPrecepted and supervised the main author throughout the publication process, Gave suggestions for revisions of the paper.

\section{ETHICS APPROVAL AND CONSENT TO PARTICIPATE}

Not applicable.

\section{ACKNOWLEDGMENT}

The authors thank the NEMA Research Team for their help and support with the preparation of this manuscript. In particular, the corresponding author would like to give a token of gratitude to Jo Ann LeQuang, the Director of Editorial Services, Medical Informatics for NEMA Research for her guidance as a mentor throughout the entire publication process. The authors would also like to thank all the peer reviewers for their opinions and suggestions.

\section{FUNDING}

This research received no external funding.

\section{CONFLICT OF INTEREST}

The authors declare no conflict of interest.

\section{REFERENCES}

[1] Hoti K, Jakupi A, Hetemi D, Raka D, Hughes J, Desselle S. Provision of community pharmacy services during COVID-19 pandemic: a cross 
sectional study of community pharmacists' experiences with preventative measures and sources of information. International Journal of Clinical Pharmacy. 2020; 42: 1197-1206.

[2] Merks P, Jakubowska M, Drelich E, Świeczkowski D, Bogusz J, Bilmin $\mathrm{K}$, et al. The legal extension of the role of pharmacists in light of the COVID-19 global pandemic. Research in Social and Administrative Pharmacy. 2020; 17: 1807-1812.

[3] Erku DA, Belachew SA, Abrha S, Sinnollareddy M, Thomas J, Steadman $\mathrm{KJ}$, et al. When fear and misinformation go viral: Pharmacists' role in deterring medication misinformation during the 'infodemic' surrounding COVID-19. Research in Social and Administrative Pharmacy. 2021; 17: 1954-1963.

[4] Strand MA, Bratberg J, Eukel H, Hardy M, Williams C. Community Pharmacists' Contributions to Disease Management During the COVID19 Pandemic. Preventing Chronic Disease. 2020; 17: E69.

[5] Di Girolamo N, Meursinge Reynders R. Characteristics of scientific articles on COVID-19 published during the initial 3 months of the pandemic. Scientometrics. 2020; 125: 795-812.

[6] Elbeddini A, Prabaharan T, Almasalkhi S, Tran C. Pharmacists and COVID-19. Journal of Pharmaceutical Policy and Practice. 2020; 13: 36.

[7] Hayden JC, Parkin R. The challenges of COVID-19 for community pharmacists and opportunities for the future. Irish Journal of Psychological Medicine. 2020; 37: 198-203.

[8] Karasneh R, Al-Azzam S, Muflih S, Soudah O, Hawamdeh S, Khader Y. Media's effect on shaping knowledge, awareness risk perceptions and communication practices of pandemic COVID-19 among pharmacists. Research in Social and Administrative Pharmacy. 2021; 17: 1897-1902.

[9] Johnston K, O'Reilly CL, Cooper G, Mitchell I. The burden of COVID-19 on pharmacists. Journal of the American Pharmacists Association. 2021; 61: e61-e64.

[10] Zheng S, Yang L, Zhou P, Li H, Liu F, Zhao R. Recommendations and guidance for providing pharmaceutical care services during COVID-19 pandemic: a China perspective. Research in Social and Administrative Pharmacy. 2021; 17: 1819-1824.

[11] Li H, Zheng S, Liu F, Liu W, Zhao R. Fighting against COVID-19: Innovative strategies for clinical pharmacists. Research in Social and Administrative Pharmacy. 2021; 17: 1813-1818.

[12] Song Z, Hu Y, Zheng S, Yang L, Zhao R. Hospital pharmacists' pharmaceutical care for hospitalized patients with COVID-19: Recommendations and guidance from clinical experience. Research in Social and Administrative Pharmacy. 2021; 17: 2027-2031.

[13] Mallhi TH, Liaqat A, Abid A, Khan YH, Alotaibi NH, Alzarea AI, et al. Multilevel Engagements of Pharmacists During the COVID-19 Pandemic: The Way Forward. Frontiers in Public Health. 2020; 8: 561924.

[14] Baye AM, Ababu A, Bayisa R, Abdella M, Diriba E, Wale M, et al. Perspectives of compounding pharmacists on alcohol-based hand sanitizer production and utilization for COVID-19 prevention in Addis Ababa, Ethiopia: A descriptive phenomenology study. PLoS ONE. 2021; 16: e0250020.

[15] Bragazzi NL, Mansour M, Bonsignore A, Ciliberti R. The Role of Hospital and Community Pharmacists in the Management of COVID19: Towards an Expanded Definition of the Roles, Responsibilities, and Duties of the Pharmacist. Pharmacy. 2020; 8: 140.

How to cite this article: Bryan Zhang, Justina Refela, Frank Breve, Peter Magnusson, Joseph Pergolizzi. COVID19 pharmacy student perceptions: pharmacists' impact during the COVID-19 pandemic. Signa Vitae. 2022;18(1):62-67. doi: $10.22514 /$ sv.2021.208. 